Scientific Paper

\title{
A Monte Carlo investigation of the dose distribution for new l-125 Low Dose Rate brachytherapy source in water and in different media
}

\author{
Zeinab FARDI ${ }^{1}$, Payvand TAHERPARVAR ${ }^{1, a}$ \\ ${ }^{I}$ Department of Physics, Faculty of Science, University of Guilan, Postal Code 4193833697, Rasht, Iran \\ ${ }^{a}$ E-mail address: p.taherparvar@gmail.com,p.taherparvar@guilan.ac.ir
}

(received 23 October 2018; accepted 8 January 2019)

\begin{abstract}
Permanent and temporary implantation of I-125 brachytherapy sources has become an official method for the treatment of different cancers. In this technique, it is essential to determine dose distribution around the brachytherapy source to choose the optimal treatment plan. In this study, the dosimetric parameters for a new interstitial brachytherapy source I125 (IrSeed-125) were calculated with GATE/GEANT4 Monte Carlo code. Dose rate constant, radial dose function and 2D anisotropy function were calculated inside a water phantom (based on the recommendations of TG-43U1 protocol), and inside several tissue phantoms around the IrSeed-125 capsule. Acquired results were compared with MCNP simulation and experimental data. The dose rate constant of IrSeed-125 in the water phantom was about $1.038 \mathrm{cGy} \cdot \mathrm{h}^{-1} \mathrm{U}^{-1}$ that shows good consistency with the experimental data. The radial dose function at $0.5,0.9,1.8,3$ and $7 \mathrm{~cm}$ radial distances were obtained as $1.095,1.019,0.826,0.605$, and 0.188 , respectively. The results of the IrSeed-125 is not only in good agreement with those calculated by other simulation with MCNP code but also are closer to the experimental results. Discrepancies in the estimation of dose around IrSeed-125 capsule in the muscle and fat tissue phantoms are greater than the breast and lung phantoms in comparison with the water phantom. Results show that GATE/GEANT4 Monte Carlo code produces accurate results for dosimetric parameters of the IrSeed-125 LDR brachytherapy source with choosing the appropriate physics list. There are some differences in the dose calculation in the tissue phantoms in comparison with water phantom, especially in long distances from the source center, which may cause errors in the estimation of dose around brachytherapy sources that are not taken account by the TG43-U1 formalism.
\end{abstract}

Key words: brachytherapy; dosimetric parameters; TG-43U1; IrSeed-125; Monte Carlo.

\section{Introduction}

Brachytherapy is an effective method in clinical radiotherapy in which an encapsulated radionuclide as the radiation source is placed within or close to a tumor inside the patient's body [1]. The main advantages of brachytherapy technique are to eliminate cancer cells, while normal tissues receive the minimal damage. Today, the low-energy photon emitting radionuclides such as Iodine-125 (I-125), Palladium-103 (Pd103) and Cesium-131 (Cs-131) are widely used in brachytherapy for treatment of certain types of cancer, such as malignant tumors in the eye, prostate cancer, cancers of the cervix, and malignant brain tumors [2]. I-125 is the most commonly used as low dose rate (LDR) source for local treatment brachytherapy. It has a half-life of 59.431 days and it decays by electron capture to excited state Tellurium-125. The Tellurium-125 then emits gamma radiation the maximum energy of $35.5 \mathrm{keV}$ to reach steady state [3]. The Iodine-125 brachytherapy capsules are available in different physical and geometrical features, such as Amersham model 6711, Amersham model 6702, Inter-source125, Best Medical Model 2301, PharmaSeed model BT-125, IsoAid Advantage, I125-SL, etc. [4-7]. Recently, a new design of iodine-125 source, which is known as IrSeed-125, is produced in Nuclear Science and Technology Research Institute of Atomic Energy Organization of Iran (AEOI) for use in interstitial brachytherapy applications [8]. According to the recommendation of the American Association of Physicists in Medicine (AAPM) Task group 43 (TG-43U1) protocol, determination of the dosimetric parameters of the brachytherapy sources is very important before clinical applications. These dosimetric parameters consist of dose rate constant, $\Lambda$, radial dose function, $g(r), 2 D$ anisotropy function, $\mathrm{F}(\mathrm{r}, \theta)$, and $1 \mathrm{D}$ anisotropy function.

In this way, experimental measurements and simulation techniques were done to calculate the dosimetric parameters for the solid brachytherapy sources. In the last several years, simulations based on the Monte Carlo techniques have become a powerful and flexible tool for determination of dosimetric parameters of the brachytherapy sources. Many Monte Carlo codes, such as MCNP [9], FLUKA [10], GATE [11], GEANT4 [12], and EGS [13], have been developed and applied for brachytherapy research. 


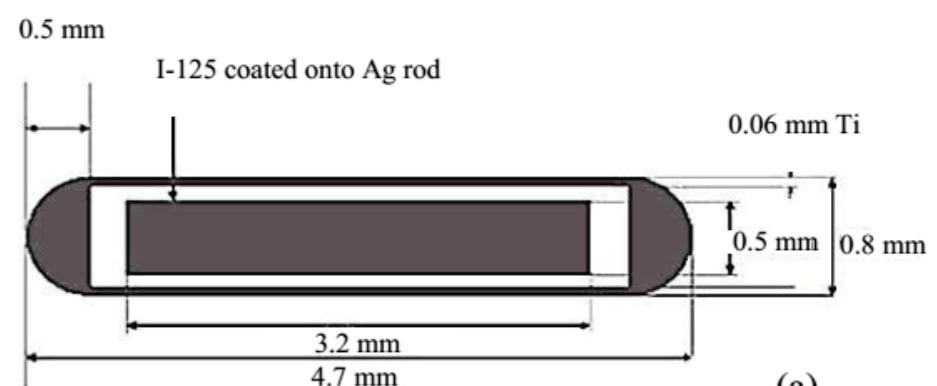

(a)

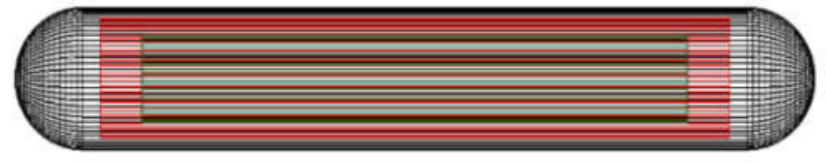

(b)

Figure 1. (a) Geometric diagram of the IrSeed-125 brachytherapy source [17] and (b) simulated capsule by GATE 8.1 code.

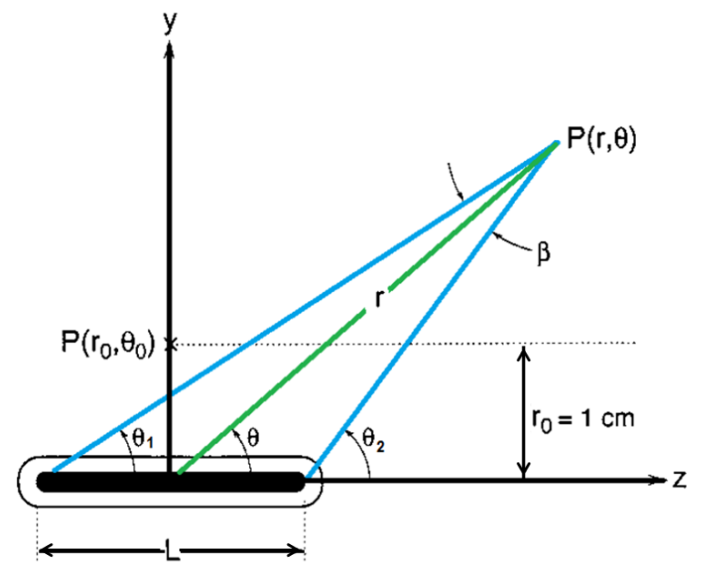

Figure 2. The geometry conventions for dose rate calculations in the TG-43 protocol.

Various studies currently use different Monte Carlo codes for evaluation of dosimetry parameters of the old and new designs of I-125 brachytherapy sources, which are as following: Meigooni et al. [14] determined the TG-43 recommended dosimetric characteristics of InterSource125 Iodine brachytherapy source theoretically with PTRAN Monte Carlo code and experimentally. Rodriguez et al. [15] estimated dosimetry parameters I-125 brachytherapy seed Model 6711 with PENELOPE Monte Carlo code. Thiam et al. [11] published the TG-43 dosimetry parameters for the different models of seed I-125 using GATE Monte Carlo simulation platform. Mark J. Rivard determined the dosimetry parameters for the I-125 brachytherapy source model 9011 and 6711 using MCNP Monte Carlo code [16]. Lohrabian et al. experimentally determined the dosimetric parameters of IrSeed-125 source using thermoluminescent dosimetry TLD-100 (LiF:Mg) [8] and Baghani et al. [17] determined the dosimetric parameters of IrSeed-125 source with MCNP Monte Carlo code.

The aim of this study is to determine the dosimetric parameters of the new IrSeed-125 solid source according to TG-43U1 protocol in the water phantom. The results of the GATE/GEANT4 simulation has been compared and validated with the previous Monte Carlo simulation and experimental measurements, with choosing the appropriate physics list. On the other hand, TG-43U1 protocol does not consider tissue atomic composition and density as scattering medium. Monte Carlo calculations for the IrSeed-125 dosimetry in the different tissues are still unknown. Therefore, we determine the dosimetric parameters of this brachytherapy source in the several tissue simulating phantoms using GATE/GEANT4 Monte Carlo code. Indeed, because the attenuation coefficient of the sources in the water is different from that of different tissues, the effects of the muscle, breast, lung and fat tissue were studied on the radial dose function of the IrSeed-125 brachytherapy source and the results were compared with the obtained results in the water phantom.

\section{Materials and methods}

\section{Source characteristics}

IrSeed-125 brachytherapy source, Figure 1a, which is manufactured by the AEOI, were simulated in this study by use of the GATE 8.1 Monte Carlo code, as shown in Figure 1b. This source, consist of a silver cylindrical marker with 0.025 $\mathrm{cm}$ radius and $3.2 \mathrm{~mm}$ length. I-125 isotope is uniformly deposited on the marker with the thickness equal to $1 \mu \mathrm{m}$. The silver cylindrical marker is encapsulated within a titanium cylinder tube of $0.47 \mathrm{~cm}$ length, $0.08 \mathrm{~cm}$ diameter and 0.006 $\mathrm{cm}$ thickness in top and bottom and $0.4 \mathrm{~mm}$ radius at both semispherical ends. The space between the marker and titanium encapsulated is filled with air.

\section{Dosimetric parameters}

Following the recommendations of the AAPM Task Group No.43 [3] updated in 2004 [18], introduced a formalism for calculation of 2D dose distribution around brachytherapy sources. The dose-rate at point $(r, \theta)$ indicated in Equation 1:

$\dot{D}(r, \theta)=S_{k} \cdot \Lambda \cdot \frac{G_{l}(r, \theta)}{G_{l}\left(r_{0}, \theta_{0}\right)} \cdot g_{l}(r) \cdot F(r, \theta)$ Eq. 1

where $\dot{\mathrm{D}}(\mathrm{r}, \theta)$ is the dose rate at the distance $\mathrm{r}(\mathrm{cm})$ from a brachytherapy capsule, $\theta$ is the polar angle defining the point of interest, $S_{k}$ is the air-kerma strength, $\Lambda$ is the dose rate constant. Furthermore, $\mathrm{G}(\mathrm{r}, \theta)$ and $\mathrm{G}\left(\mathrm{r}_{0}, \theta_{0}\right)$ are the geometry factors in point of interest around the source and reference point $\left(r_{0}=1 \mathrm{~cm}, \theta_{0}=90^{\circ}\right)$, respectively [18]. The geometric system used for the dosimetric calculations are shown in Figure 2. 
According to the TG-43 (U1) protocol, dose rate constant is obtained by dividing dose rate at the reference point $\left(\mathrm{r}_{0}=1 \mathrm{~cm}\right.$, $\theta_{0}=\pi / 2$ ) into air kerma strength of the source.

$\Lambda=\frac{\dot{D}\left(r_{0}, \Theta_{0}\right)}{S_{k}}$

To calculate the air kerma strength of the source $\left(S_{k}\right)$, first the air kerma rate was calculated within an air filled spherical scoring cell with radius of $2 \mathrm{~mm}$ that was located at $50 \mathrm{~cm}$ distance from the source center along its transverse axis in vacuum phantom. Then, this parameter multiplied by the square of the distance $\left(\mathrm{d}^{2}\right)$ as following:

$S_{k}=\dot{K}(d) d^{2} \& d=50 \mathrm{~cm}$

Eq. 3

where $\dot{K}(d)$ is air kerma rate at a distance of d. $5 \times 10^{8}$ particles were followed to obtain least statistical uncertainty. To calculate the radial dose function, $\mathrm{g}(\mathrm{r})$, rings with $0.4 \mathrm{~mm}$ thicknesses were located at 0.1 to $7 \mathrm{~cm}$ distance from the source center along its transverse axis. Then, $g(r)$ was calculated according to Equation 4.

$g(r)=\frac{\dot{D}\left(r, \theta_{0}\right)}{\dot{D}\left(r_{0}, \theta_{0}\right)} \frac{G\left(r_{0}, \Theta_{0}\right)}{G\left(r, \theta_{0}\right)}$

Eq. 4

The geometric systems used to calculate $\mathrm{g}(\mathrm{r})$ is presented in Figure 3a. The anisotropy function of IrSeed-125 source was measured at distances of $0.5,1,2,3,5,7 \mathrm{~cm}$ from the source center using rings with $0.4 \mathrm{~mm}$ thicknesses at different angle relative to the source axis and it was calculated according to Equation 5. The system used to calculate anisotropy function $\mathrm{F}(r, \theta)$ is described in Figure 3b.

$F(r, \theta)=\frac{\dot{D}(r, \theta)}{\dot{D}\left(r, \theta_{0}\right)} \frac{G\left(r, \theta_{0}\right)}{G(r, \theta)}$

The $G(r, \theta)$ geometry function only dependent on the source dimensions and ignoring photon absorption and scattering in the source structure. For a line source approximation, it is defined as:

$G(r, \theta)=\left\{\begin{array}{c}\frac{\beta}{L r \sin \theta}, \theta \neq 0^{\circ} \\ \left(r^{2}-L^{2} / 4\right)^{-1}, \theta=0^{\circ}\end{array}\right.$

where $\mathrm{L}$ is called source active length and is assumed to be 3.2 $\mathrm{mm}$. In addition, $\beta$ angle is shown in the Figure 2.

\section{Monte Carlo simulation}

In this work, simulations were performed using version 8.1 of the GATE (GEANT4 Application for Emission Tomography) Monte Carlo code. In the beginning, this platform, based on several hundred $\mathrm{C}++$ classes, was designed to meet specific needs of the simulations of nuclear medical imaging and many studies were performed in this field [11]. GATE combines the advantages of the GEANT4 simulation toolkit well-validated physics models, sophisticated geometry description, and powerful visualization and $3 \mathrm{D}$ rendering tools with original features specific to emission tomography [19] as well as radiation therapy [11].
In our simulation, we used GATE 8.1 code to determine the dosimetric parameters of the IrSeed-125 brachytherapy source in a $20 \mathrm{~cm}$ radius spherical water phantom according to the recommendation of the AAPM. IrSeed-125 brachytherapy source was located in the center of the phantom and radial dose function and 2D anisotropy function were calculated. According to TG-43 (U1) recommendations, reference dosimetry media was considered degassed water with a mass density of $0.998 \mathrm{~g} / \mathrm{cm}^{3}$. To calculate air kerma strength source $\left(\mathrm{S}_{\mathrm{k}}\right)$ the capsule was located at the center of vacuum phantom. I-125 photon spectrum was taken from NCRP58 [20]. This photons spectrum is shown in Table 1. On the other hand, since the attenuation coefficient of the sources in the water phantom is different from that of various tissues (lung, fat, breast, and muscle) $[21,22]$, the effects of the various tissues on the radial dose function of the I-125 brachytherapy source were investigated in this study. The compositions of various tissues used in the simulations are based on the ICRU (International Commission on Radiation Units and Measurements) report 44 [23], as shown in Table 2.

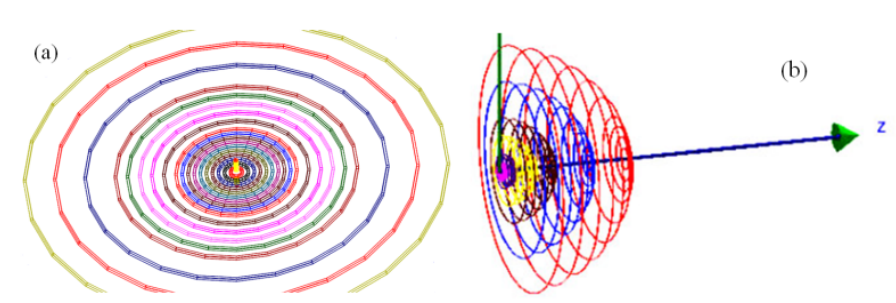

Figure 3. Geometric system used to calculate $g(r)$ (a) and $F(r, \theta)$ (b) in the GATE 8.1 code

Table 1. Photon spectrum of I-125, according to the NCRP58 [20].

\begin{tabular}{cc}
\hline \hline Energy (keV) & Number of photons in each decay \\
\hline 3.77000 & 0.150 \\
27.2017 & 0.397 \\
27.4723 & 0.741 \\
31.0000 & 0.257 \\
35.4919 & 0.067 \\
\hline \hline
\end{tabular}

Table 2. Compositions of the breast, lung, and muscle tissues based on the ICRU Report 44 [23].

\begin{tabular}{ccccc}
\hline \hline & Breast & Lung & Fat & Muscle \\
\hline $\mathrm{H}$ & 10.6 & 10.3 & 11.4 & 10.2 \\
$\mathrm{C}$ & 33.2 & 10.5 & 59.8 & 14.3 \\
$\mathrm{~N}$ & 3.0 & 3.1 & 0.7 & 3.4 \\
$\mathrm{O}$ & 52.7 & 74.9 & 27.8 & 71.0 \\
$\mathrm{Na}$ & 0.1 & 0.2 & 0.1 & 0.1 \\
$\mathrm{Mg}$ & - & - & 0.1 & - \\
$\mathrm{Cl}$ & 0.1 & 0.3 & 0.1 & 0.1 \\
$\mathrm{P}$ & 0.1 & 0.2 & - & 0.3 \\
$\mathrm{~S}$ & 0.2 & 0.3 & - & 0.1 \\
$\mathrm{~K}$ & - & 0.2 & - & 0.4 \\
Density $\left(\mathbf{g r} / \mathrm{cm}^{\mathbf{3}}\right)$ & 1.02 & 1.05 & 0.95 & 1.05 \\
\hline \hline
\end{tabular}




\section{Results and discussion}

\section{Dose rate constant}

The dose rate constant, $\Lambda$, value was obtained by use of the airkerma strength in the vacuum medium using the GATE 8.1 simulation with emstandard_opt3 physics list, which was about $1.038 \mathrm{cGy} \cdot \mathrm{h}^{-1} \mathrm{U}^{-1}$. The acquired dose rate constant is in good agreement with the Baghani et al. [17] calculation, 0.983 $\mathrm{cGy} \cdot \mathrm{h}^{-1} \mathrm{U}^{-1}$, which have used the MCNP code. The difference between calculated data by GATE and MCNP simulation was about $5.5 \%$. We also present dose rate constant results for some common models of I-125 brachytherapy sources in Table 3. It appears that the results of the dose rate constant are close to each other for different type of I-125 seeds. As seen in this table, the dose rate constant of model 6711 and 6702 was reported to be 0.965 and $1.036 \mathrm{cGy} \cdot \mathrm{h}^{-1} \mathrm{U}^{-1}$, respectively, which are acquired by use of the average of Monte Carlo simulation and measurement [16]. Moreover, the dose rate constant has been reported to be 1.01 and $0.961 \mathrm{cGy} \cdot \mathrm{h}^{-1} \mathrm{U}^{-1}$ by Sowards and Meigooni [24] and Meigooni et al. [25] using the Monte Carlo simulation and measurement for Best model 2301 brachytherapy source. The result of our calculation is close to the other I-125 brachytherapy sources. The differences between Monte Carlo reported data even for the same source type is related to some difference in nuclear data libraries used by MCNP, GEANT4, PENELOPE, FLUKA and so on, which can affect the ability of each Monte Carlo code.

Table 3. Comparison of the IrSeed-125 dose rate constant to that of some other commercially available sources.

\begin{tabular}{|c|c|c|c|}
\hline Source model & Method & Medium & Dose rate constant $\left(\mathrm{cGy} \mathrm{h}^{-1} \mathrm{U}^{-1}\right)$ \\
\hline IrSeed125 (This Work) & Monte Carlo simulation & Water & 1.038 \\
\hline IrSeed125 (Baghani, et al.2016) & Monte Carlo simulation & Water & 0.983 \\
\hline IrSeed125 (Lohrabian, et al.2013) & Measurement & Plexiglass & 0.965 \\
\hline BestModel2301 (Sowards and Meigooni, 2002) & Monte Carlo simulation & Water & 1.01 \\
\hline InterSource125 (Meigooni, et al. 2002) & Monte Carlo simulation & Water & 1.013 \\
\hline Model6711 (Williamson, 1991) & Monte Carlo simulation & Water & 0.877 \\
\hline Model6702 (Rivard, et al. 2004) & Average of Monte Carlo simulation and measurement & Water & 1.036 \\
\hline Model6711 (Rivard, et al. 2004) & Average of Monte Carlo simulation and measurement & Water & 0.965 \\
\hline BestModel2301 (Rivard, et al. 2004) & Average of Monte Carlo simulation and measurement & Water & 1.018 \\
\hline BestModel2301 (Meigooni, et al. 2000) & Measurement & Solid Water & 0.961 \\
\hline
\end{tabular}

Table 4. Radial dose function of IrSeed-125 source obtained from different study

\begin{tabular}{|c|c|c|c|}
\hline $\begin{array}{l}\text { Radial distance } \\
\qquad(\mathrm{cm})\end{array}$ & $\begin{array}{c}\text { GATE } \\
\text { (This work) }\end{array}$ & $\begin{array}{c}\text { MCNP; } \\
\text { Baghani et al. } \\
(2016)[17]\end{array}$ & $\begin{array}{c}\text { Measurement; } \\
\text { Lohrabian et al. } \\
\text { (2013) [8] }\end{array}$ \\
\hline 0.1 & 1.118 & 1.071 & - \\
\hline 0.2 & 1.147 & 1.083 & - \\
\hline 0.3 & 1.136 & 1.078 & - \\
\hline 0.4 & 1.123 & 1.074 & - \\
\hline 0.5 & 1.095 & 1.063 & 1.011 \\
\hline 0.6 & 1.082 & 1.053 & - \\
\hline 0.7 & 1.052 & 1.041 & - \\
\hline 0.8 & 1.033 & 1.028 & - \\
\hline 0.9 & 1.019 & 1.014 & - \\
\hline 1.0 & 1.000 & 1.000 & 1.000 \\
\hline 1.2 & 0.948 & 0.967 & - \\
\hline 1.4 & 0.906 & 0.934 & - \\
\hline 1.6 & 0.876 & 0.900 & - \\
\hline 1.8 & 0.826 & 0.865 & - \\
\hline 2 & 0.784 & 0.830 & 0.803 \\
\hline 2.5 & 0.682 & 0.738 & - \\
\hline 3 & 0.605 & 0.657 & 0.612 \\
\hline 3.5 & 0.537 & 0.575 & - \\
\hline 4 & 0.459 & 0.505 & 0.478 \\
\hline 4.5 & 0.407 & 0.441 & - \\
\hline 5 & 0.346 & 0.384 & 0.352 \\
\hline 5.5 & 0.307 & 0.327 & - \\
\hline 6 & 0.259 & 0.283 & 0.270 \\
\hline 6.5 & 0.208 & 0.230 & - \\
\hline 7 & 0.188 & 0.182 & 0.208 \\
\hline
\end{tabular}

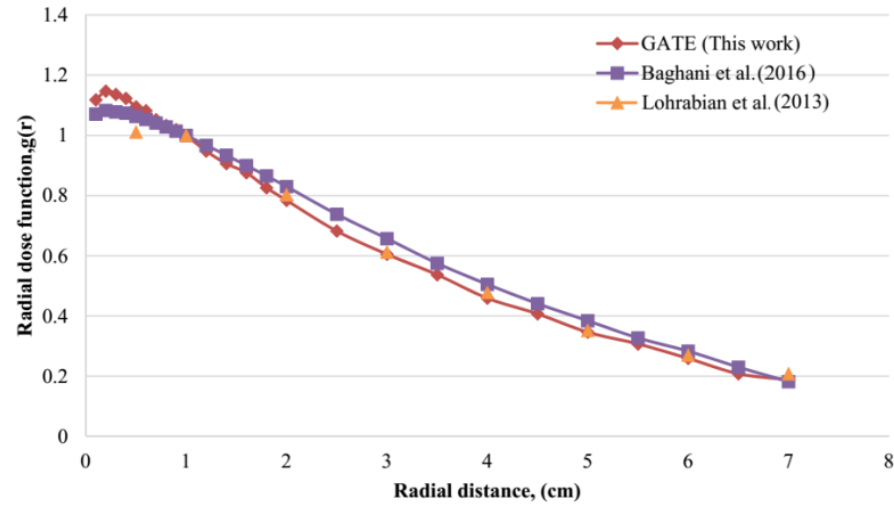

Figure 4. Comparison of the radial dose function obtained by GATE 8.1 simulation results and other experimental and theoretical results for the IrSeed-125 source.

\section{Radial dose function}

Values of the radial dose function for IrSeed-125 in the degassed water phantom was calculated for distances from 1 to $7 \mathrm{~cm}$ from the source center by GATE 8.1 code. These results are indicated in Table 4. Furthermore, the comparison between the theoretical and experimental radial dose function is shown in Figure 4. The mean difference between our results and those of experiments [8] and MCNP simulation [17] were about $4.4 \%$ and $5.0 \%$, respectively. 


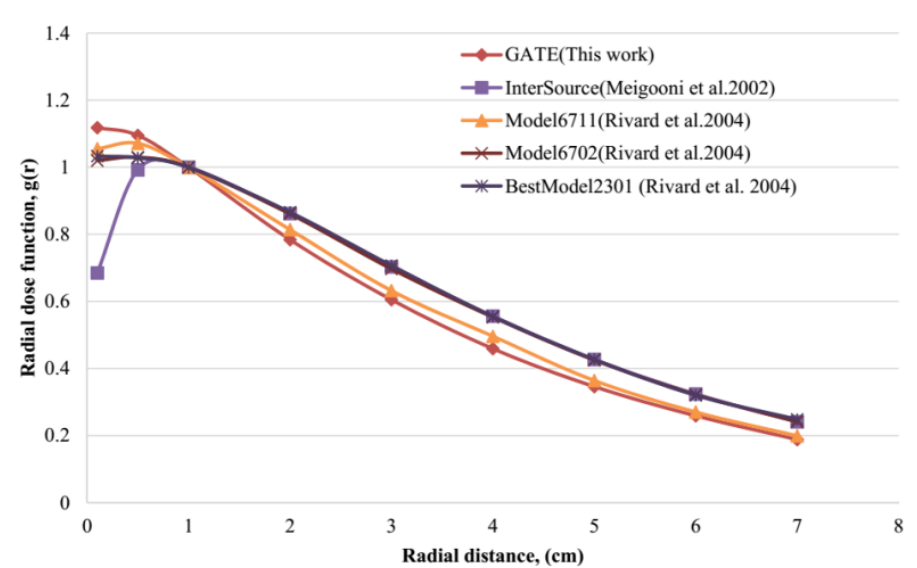

Figure 5. Comparison between the radial dose function of the simulated IrSeed-125 and other available sources

Table 5. Anisotropy function calculated for the IrSeed-125.

\begin{tabular}{ccccccc}
\hline \hline $\begin{array}{c}\text { Angle/r } \\
(\mathbf{c m})\end{array}$ & $\mathbf{0 . 5}$ & $\mathbf{1}$ & $\mathbf{2}$ & $\mathbf{3}$ & $\mathbf{5}$ & $\mathbf{7}$ \\
\hline 0 & 0.214 & 0.298 & 0.267 & 0.354 & 0.662 & - \\
5 & 0.297 & 0.402 & 0.517 & 0.524 & 0.606 & 0.566 \\
10 & 0.368 & 0.445 & 0.550 & 0.604 & 0.619 & 0.652 \\
15 & 0.491 & 0.560 & 0.635 & 0.676 & 0.722 & 0.699 \\
20 & 0.611 & 0.644 & 0.713 & 0.751 & 0.806 & 0.845 \\
30 & 0.799 & 0.795 & 0.830 & 0.855 & 0.823 & 0.917 \\
40 & 0.923 & 0.888 & 0.909 & 0.942 & 0.886 & 0.916 \\
50 & 1.047 & 0.963 & 0.995 & 0.973 & 0.946 & 1.041 \\
60 & 1.018 & 1.007 & 1.034 & 1.022 & 1.018 & 1.034 \\
70 & 1.019 & 1.027 & 1.036 & 1.022 & 0.984 & 1.069 \\
80 & 1.006 & 1.022 & 1.032 & 1.039 & 1.010 & 1.060 \\
90 & 1.000 & 1.000 & 1.000 & 1.000 & 1.000 & 1.000 \\
\hline \hline
\end{tabular}

Figure 5 shows the Monte Carlo calculated radial dose function obtained in the water phantom at different distances from the source center. In this figure, the GATE results are compared to the data obtained by Meigooni et al. [14], and Rivard et al. [18].

According to Figure 5 results, there is a good agreement between our simulation results for radial dose function of IrSeed-125 and previously reported data. The simulated radial dose function was determined by fitting a $5^{\text {th }}$ order polynomial function to the acquired data, as follows:

$g(r)=a_{0}+a_{1} r+a_{2} r^{2}+a_{3} r^{3}+a_{4} r^{4}+a_{5} r^{5}$

where $a_{0}, a_{1}, a_{2}, a_{3}, a_{4}, a_{5}$ are coefficients of the radial dose function which were obtained as $1.1515,-0.0594,-0.1348$, 0.0487, 0.0069, and 0.0004, respectively.

\section{Anisotropy function}

Table 5 shows the calculated anisotropy function values at radial distances of $0.5,1,2,3,5,7 \mathrm{~cm}$ for angles between $0^{\circ}$ and $90^{\circ}$ in increments of 5 and 10 degrees in the short and large distances, respectively. Moreover, Figure 6 shows a comparison of the anisotropy function of the IrSeed-125
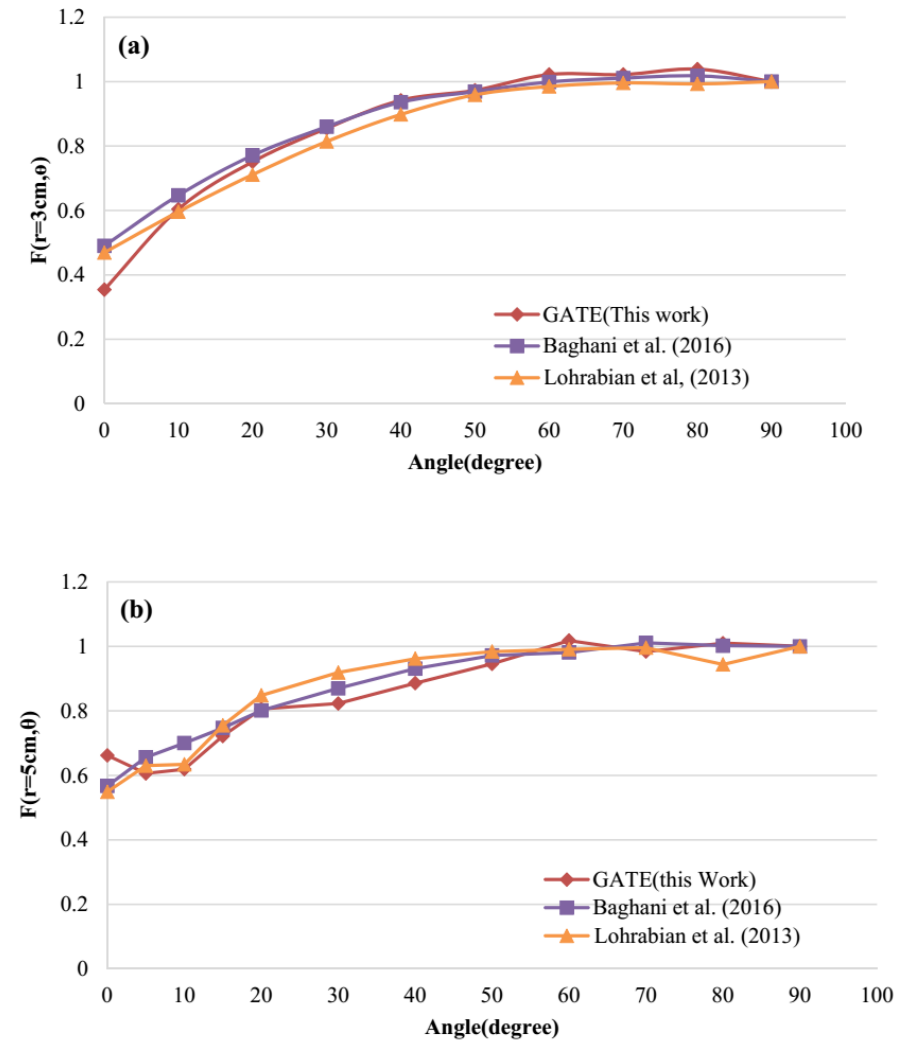

Figure 6. Calculated anisotropy function of the IrSeed-125 compared to the results of Baghani et al. [13] and Lohrabian et al. [8] at two radial distances; (a) $3 \mathrm{~cm}$ and (b) $5 \mathrm{~cm}$.

source, which is calculated by GATE code, with the experimental data measured by Lohrabian et al. [8] and the Baghani et al. simulation results [17], which is calculated by the MCNP code, at two radial distances of 3 (Figure 6a) and 5 $\mathrm{cm}$ (Figure 6b). Results show that the average difference between calculated data of GATE 8.1 with MCNP simulation results is about $5.65 \%(5.46 \%)$ at $3 \mathrm{~cm}(5 \mathrm{~cm})$ distance. These differences with experimental data measured (with TLD) are about $5.72 \%$ and $6.29 \%$ at $3 \mathrm{~cm}$ and $5 \mathrm{~cm}$ distances, respectively. Furthermore, the comparison of the simulated anisotropy function of IrSeed-125 to that of other available sources at different radial distances are presented in Figure 7 (a to d), at different radial distances of $1-5 \mathrm{~cm}$. As it can be seen in these diagrams, there is a good agreement between the GATE 8.1 results and those related to other commercial sources, especially at angles larger than $20^{\circ}$. The differences at angles less than $20^{\circ}$ might be due to the differences in designing the source geometry, composition and density of the materials, as well as some difference in the nuclear data libraries of different codes. 

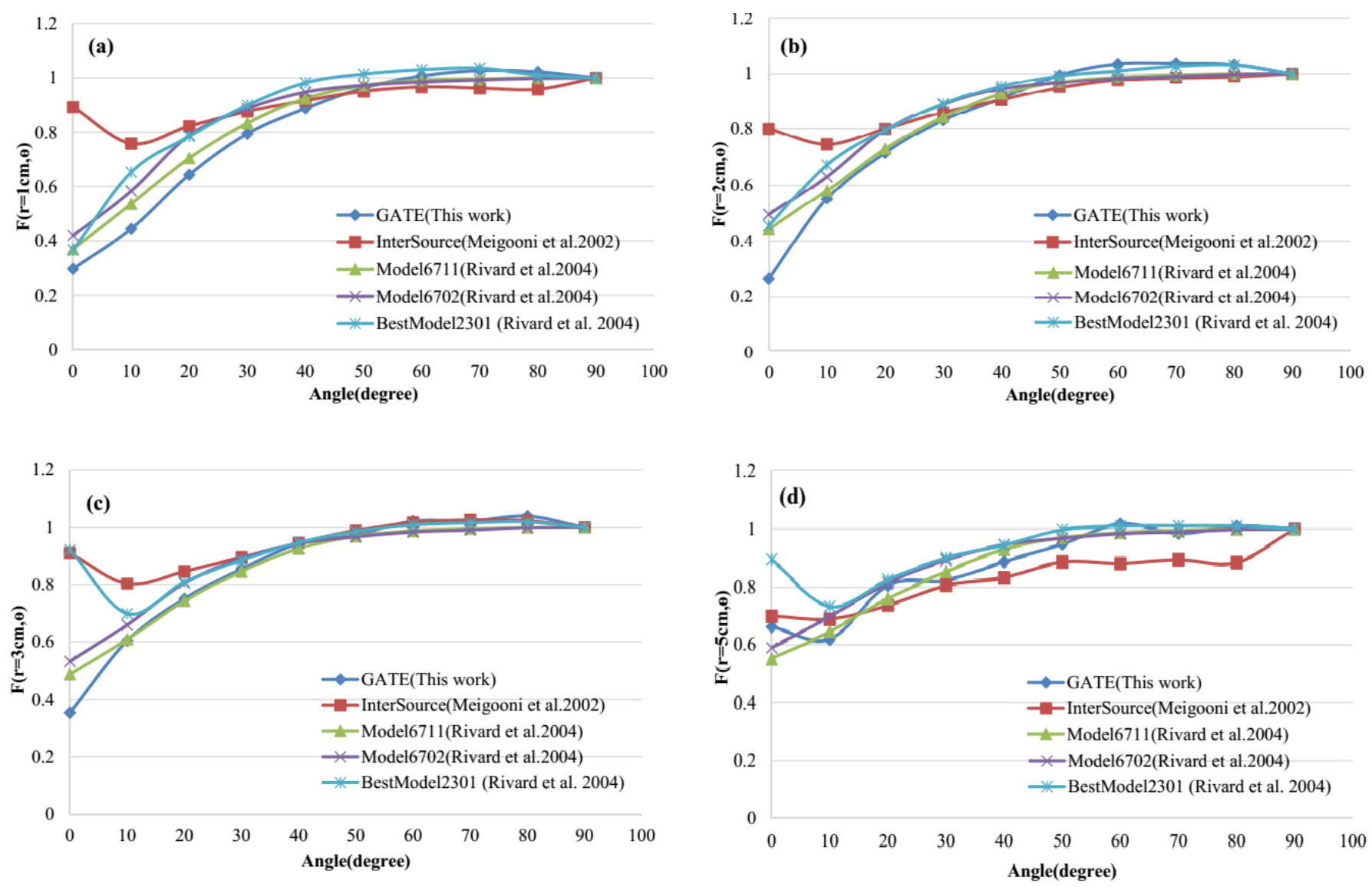

Figure 7. Comparison between the simulated anisotropy function of the IrSeed-125 and other available sources at different radial distances; $1 \mathrm{~cm}(\mathrm{a}) ; 2 \mathrm{~cm}(\mathrm{~b}) ; 3 \mathrm{~cm}(\mathrm{c})$, and $5 \mathrm{~cm}(\mathrm{~d})$.

Table 6. Radial dose function, $g(r)$, in different media calculated by GATE code.

\begin{tabular}{cccccc}
\hline $\begin{array}{c}\text { Radial } \\
\text { distance } \\
\text { (cm) }\end{array}$ & Water & Fat & Muscle & Breast & Lung \\
\hline 0.1 & 1.118 & 0.984 & 1.151 & 1.067 & 1.158 \\
0.2 & 1.147 & 1.004 & 1.177 & 1.096 & 1.181 \\
0.3 & 1.136 & 1.008 & 1.160 & 1.100 & 1.165 \\
0.4 & 1.123 & 1.009 & 1.137 & 1.086 & 1.141 \\
0.5 & 1.095 & 1.003 & 1.117 & 1.076 & 1.120 \\
0.6 & 1.082 & 1.006 & 1.093 & 1.072 & 1.096 \\
0.7 & 1.052 & 1.004 & 1.076 & 1.045 & 1.079 \\
0.8 & 1.033 & 1.001 & 1.052 & 1.028 & 1.050 \\
0.9 & 1.019 & 1.010 & 1.025 & 1.025 & 1.026 \\
1.0 & 1.000 & 1.000 & 1.000 & 1.000 & 1.000 \\
1.2 & 0.948 & 0.977 & 0.957 & 0.972 & 0.960 \\
1.4 & 0.906 & 0.979 & 0.915 & 0.946 & 0.903 \\
1.6 & 0.876 & 0.941 & 0.882 & 0.907 & 0.857 \\
1.8 & 0.826 & 0.910 & 0.817 & 0.888 & 0.819 \\
2 & 0.784 & 0.915 & 0.773 & 0.853 & 0.771 \\
2.5 & 0.682 & 0.870 & 0.676 & 0.793 & 0.674 \\
3 & 0.605 & 0.811 & 0.593 & 0.685 & 0.584 \\
3.5 & 0.537 & 0.761 & 0.510 & 0.607 & 0.503 \\
4 & 0.459 & 0.708 & 0.422 & 0.550 & 0.415 \\
4.5 & 0.407 & 0.655 & 0.372 & 0.489 & 0.364 \\
5 & 0.346 & 0.584 & 0.323 & 0.417 & 0.311 \\
5.5 & 0.307 & 0.550 & 0.270 & 0.384 & 0.266 \\
6 & 0.259 & 0.494 & 0.238 & 0.339 & 0.231 \\
6.5 & 0.208 & 0.475 & 0.188 & 0.285 & 0.186 \\
7 & 0.188 & 0.411 & 0.157 & 0.263 & 0.157 \\
\hline \hline & & & & &
\end{tabular}

\section{Radial dose function in different tissue}

Brachytherapy by using I- 125 seed is used worldwide to treat a wide range of cancers in different tissues. Since the attenuation coefficient of the brachytherapy sources in the water phantom is different from that of various media, the effects of the various tissues on the radial dose function parameter of the IrSeed-125 brachytherapy source were investigated using GATE 8.1 code. In this way, the radial dose function was calculated in the fat tissue, muscle, breast, and lung with 0.95 , $1.05,1.02$ and $1.05 \mathrm{gr} / \mathrm{cm}^{3}$ densities, respectively. Table 6 shows the radial dose function of the IrSeed-125 source in different tissue phantoms which are calculated by GATE 8.1 simulations. Figure 8 shows a comparison between results of radial dose function of the IrSeed-125 obtained in the tissue phantom and that obtained in water phantom. Results show that the maximum relative difference of the radial dose function at distances smaller than $1 \mathrm{~cm}$ in the fat tissue and breast relative to water phantom are $12.5 \%$ and $4.5 \%$, respectively. With increasing distance from the source center $($ at $7 \mathrm{~cm})$, these relative differences increase and reach to $118.6 \%$ and $39.9 \%$ for the fat tissue and breast, respectively. Because the density and composition of muscle and lung are similar to the water, the results of radial dose function obtained in these media and water phantom are close to each other. 


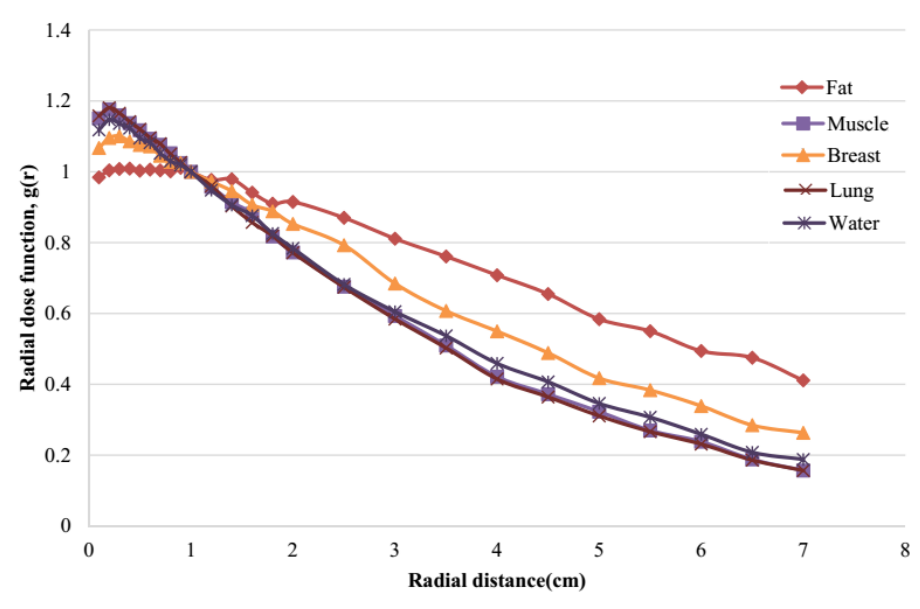

Figure 8. Radial dose function calculated by GATE 8.1 code obtained in the water, muscle, breast, lung and fat tissue.

Figure 9 presents the ratio of radial dose function obtained in the tissue phantoms to radial dose function calculated in the water. The difference between $\mathrm{g}(\mathrm{r})_{\text {Tissue }}$ and $\mathrm{g}(\mathrm{r})_{\text {Water }}$ increased with increasing the radial distance from the source center for fat tissue and breast tissue. Whereas, this difference is negligible for the lung and muscle phantoms.

\section{Summary and conclusions}

Dosimetric parameters of the new design of the I-125 brachytherapy source, which named IrSeed-125, have been investigated by using GATE/GEANT4 Monte Carlo code. Dosimetric characteristics such as; dose rate constant, radial dose function and anisotropy function of the IrSeed-125 have been determined according to the recommendation of the AAPM Task group 43 (TG-43U1) protocol. The GATE results obtained in this study show good consistency with the experimental data. The dose rate constant was calculated to be $1.034 \mathrm{cGy} \cdot \mathrm{h}^{-1} \mathrm{U}^{-1}$. This value was in good agreement with MCNP simulation results of IrSeed-125 and that of other commercially available sources. The radial dose function of the IrSeed-125 was calculated at different distances from the source center. The good agreement between present study results and previous studies shows that the correct source geometry and physics list were used in our simulation by

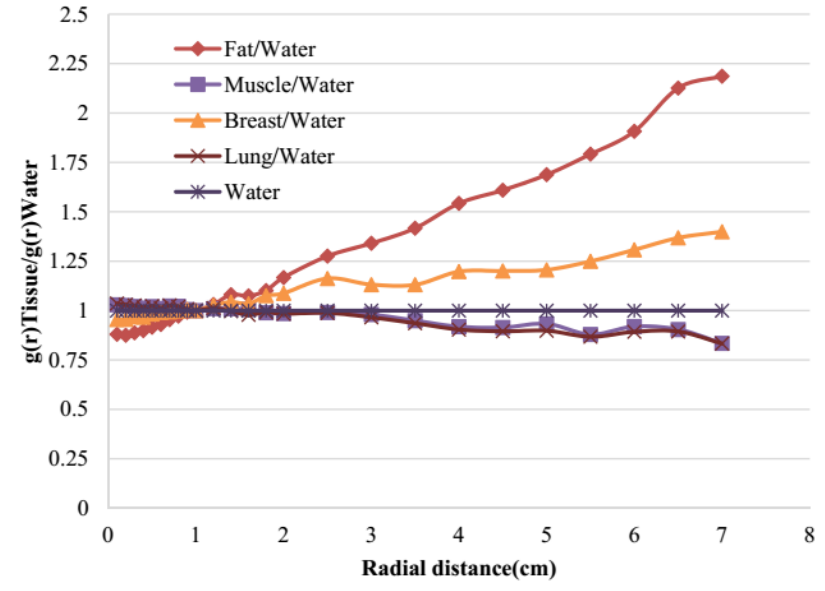

Figure 9. The ratio of $\mathrm{g}(\mathrm{r})$ in the muscle, breast, lung and fat tissue to $g(r)$ in the water.

GATE 8.1 code. The results of the radial dose function are not only in good agreement with those calculated by MCNP simulation but also are closer to the TLD experimental results. The anisotropy function of the IrSeed-125 source was calculated at angles between $0^{\circ}$ and $90^{\circ}$ at distances of $0.5,1$, 2, 3, 5, and $7 \mathrm{~cm}$ using GATE 8.1 code. Our results were in good agreement with the calculated values in the previous studies.

Dosimetry according to the TG-43U1 protocol assumes that the patient body is uniformly composed of water. Results of dose evaluation in the different phantoms consist of tissue materials show that considering the water phantom, recommended by AAPM, for evaluation of dosimetric parameters of the IrSeed-125 source, may cause errors in the estimation of dose around brachytherapy sources in the treatment planning systems. Results show that dosimetric parameters of IrSeed-125 source in breast and fat tissue are the fairly large difference in comparison with the water phantom, especially at large distances from the solid source center. The result of the validated LDR brachytherapy source could be employed to develop application of this seed in the clinical applications and dose prediction in the tumor regions and normal tissue adjacent to the tumor.

\section{References}

[1] Russell KJ, Blasko JC. Recent advances in interstitial brachytherapy for localized prostate cancer. in: Therapeutic strategies in prostate cancer. Problems in urology series. Vol. 7. 4th edition. J. B. Lippincott Co, Philadelphia; 1993: 260-278.

[2] Ghiassi-Nejad M, Jafarizadeh M, Ahmadian-Pour MR, Ghahramani AR. Dosimetric characteristics of 192Ir sources used in interstitial brachytherapy. Appl Radiat Isot. 2001;55(2):189-195.

[3] Nath R, Anderson LL, Luxton G, et al. Dosimetry of interstitial brachytherapy sources: recommendations of the AAPM Radiation Therapy Committee Task Group No. 43. American Association of Physicists in Medicine. Med Phys. 1995;22(2):209-234.

[4] Heintz BH, Wallace RE, Hevezi JM. Comparison of I-125 sources used for permanent interstitial implants. Med Phys. 2001;28(4):671-682. 
[5] Rivard MJ, Butler WM, DeWerd LA, et al. Supplement to the 2004 update of the AAPM Task Group No. 43 Report. Med Phys. 2007;34(6):2187-2205.

[6] Solberg TD, DeMarco JJ, Hugo G, Wallace RE. Dosimetric parameters of three new solid core I-125 brachytherapy source. J Appl Clin Med Phys. 2002;3(2):119-134.

[7] Wallace RE. Dosimetric characterization of a new 125Iodine brachytherapy source, model I125-SL. Proceedings of the 22nd Annual International Conference of the IEEE Engineering in Medicine and Biology Society (Cat. No.00CH37143), Chicago, IL, 2000, pp. 376-379 vol.1.

[8] Lohrabian V, Sheibani S, Aghamiri MR, et al. Determination of Dosimetric Characteristics of IrSeed I-125 Brachytherapy Source. Iran J Med Phys. 2013;10(3):109-117.

[9] Forster RA, Cox LJ, Barrett RF, et al. MCNP Version 5. Nucl Instrum Meth Phys Res B. 2004;213:82-86.

[10] Rajabi R, Taherparvar P. Monte Carlo dosimetry for a new 32P brachytherapy source using FLUKA code. J Contemp Brachytherapy. 2019. doi: $10.5114 /$ jcb.2019.83002.

[11] Thiam CO, Breton V, Donnarieix D, Habib B, et al. Validation of a dose deposited by low-energy photons using GATE/GEANT4. Phys Med Biol. 2008;53(11):3039-3055.

[12] Agostinelli S, Allison J, Amako K, et al. Geant4 - a simulation toolkit. Nucl Instrum Meth A. 2003;506(3):250-303.

[13] Nelson WR, Hirayama H, Rogers DWO. The EGS4 code system, Report SLAC-265. Stanford Linear Accelerator Center, Stanford, CA, USA, 1985.

[14] Meigooni AS, Yoe-Sein MM, Al-Otoom AY, Sowards KT. Determination of the dosimetric characteristics of InterSource125 Iodine brachytherapy source. Appl Radiat Isot. 2001;56(4):589-599.

[15] Rodríguez EAV, Alcón EPQ, Rodriguez ML, et al. Dosimetric parameters estimation using PENELOPE MonteCarlo simulation code: Model 6711 a I-125 brachytherapy seed. Appl Radiat Isot. 2005;63(1):41-48.

[16] Rivard MJ. Monte Carlo radiation dose simulations and dosimetric comparison of the model 6711 and 9011 I-125 brachytherapy sources. Med Phys. 2009;36(2):486-491.

[17] Baghani HR, Lohrabian V, Aghamiri MR, Robatjazi M. Monte Carlo Determination of Dosimetric Parameters of a New I-125 Brachytherapy Source According to AAPM TG-43 (U1) Protocol. Arch Iran Med. 2016;19(3):186-191.

[18] Rivard MJ, Corsey BM, DeWerd LA, et al. Update of AAPM Task Group No. 43 Report: a revised AAPM protocol for brachytherapy dose calculations. Med. Phys. 2004;31(3):633-674.

[19] Taherparvar P, Sadremomtaz A. Development of GATE Monte Carlo simulation for a CsI pixelated gamma camera dedicated to high resolution animal SPECT. Australas Phys Eng Sci Med. 2018;41:31-38.

[20] NCRP. A handbook of radioactivity measurements procedures; NCRP Report No. 58. Bethesda: National Council on Radiation Protection and Measurements, 1985.

[21] Badry H, Oufni L, Ouabi H, Hirayama H. A Monte Carlo investigation of the dose distribution for 60Co high dose rate brachytherapy source in water and in different media. Appl Radiat Isot. 2018;136:104-110.

[22] Ghorbani M, Salahshour F, Haghparast A, et al. Effect of tissue composition on dose distribution in brachytherapy with various photon emitting sources. J Contemp Brachytherapy. 2014;6(1):54-67.

[23] International Commission on Radiation Units and Measurements, 1989. Tissue Substitutes in Radiation Dosimetry and Measurement. ICRU Report no. 44. Bethesda, MD.

[24] Sowards KT, Meigooni AS. A Monte Carlo evaluation of the dosimetric characteristics of the Bests Model 2301 125I brachytherapy source. Appl Radiat Isot. 2002;57(3):327-333.

[25] Meigooni AS, Gearheart DM, Sowards K. Experimental determination of dosimetric characteristics of Best@ 125I brachytherapy source. Med Phys. 2000;27(9):2168-2173. 